\title{
Development of a Multiplex Polymerase Chain Reaction Method for cps Typing of Actinobacillus pleuropneumoniae Serovars 1, 2, 5, 7, and 15
}

\author{
Hiroya ITO $^{1 *}$ and Masuo SUEYOSHI ${ }^{2}$ \\ ${ }^{1}$ National Institute of Animal Health, National Agriculture and Food Research Organization (Tsukuba, \\ Ibaraki 305-0856, Japan) \\ ${ }^{2}$ Center for Animal Disease Control, University of Miyazaki (Miyazaki, Miyazaki 889-2192, Japan)
}

\begin{abstract}
We developed a multiplex polymerase chain reaction (PCR) method using primers specific to the capsular polysaccharide biosynthesis genes (cps) to type Actinobacillus pleuropneumoniae serovars 1, 2, 5,7 , and 15 . This multiplex PCR method may be useful for the typing of serovar 15 , which is recently becoming more prevalent, as well as the most prevalent serovars 1, 2, 5, and 7 in Japan.
\end{abstract}

Discipline: Animal health

Additional key words: pig, pleuropneumonia, serotyping

\section{Introduction}

Actinobacillus pleuropneumoniae is an etiologic agent of porcine pleuropneumonia that causes serious economic losses in the pig-rearing industry (Gottschalk 2012). Fifteen serovars of A.pleuropneumoniae have been identified to date, mainly based on the antigenic diversity of their capsular polysaccharides (Blackall et al. 2002, Perry et al. 1990). The prevalent local serovars vary among countries (Gottschalk 2012); in Japan, serovars 2 (59\%), 1 (28\%), $5(8 \%)$, and $7(3 \%)$ account for approximately $98 \%$ of the strains isolated from pigs (Asawa et al. 1995, Fukuyasu et al. 1991, 1996, Kume \& Nakai 1988, Morioka et al. 2006, 2008, Suzuki et al. 1989, Yoshimura et al. 2002). However, the incidence of isolated cases of serovar 15 , which is the most recently identified serovar (Blackall et al. 2002), has been recently increasing in Japan (Ito 2013, Morioka et al. 2008).

Because the virulence of strains differs depending on their serovars and differences in serovars affect the effectiveness of vaccines (Gottschalk 2012), serotyping is widely performed in veterinary diagnostic laboratories. However, cross-reactions are often observed among different serovars, for example, among serovars 1, 9, and 11 (Gottschalk 2012), serovars 3, 6, 8, and 15 (Gottschalk 2012), serovars 4 and 7 (Gottschalk 2012), and serovars 7 and 15 (Blackall et al. 2002, Koyama et al. 2007), prevent- ing the accurate and rapid typing of field strains.

In the last decade, serovar-specific polymerase chain reaction (PCR) typing methods based on the sequence variation in genes involved in capsular polysaccharide biosynthesis (cps) have been developed for serovars 1-3, 5-8, 10, 12, and 15 (Angen et al. 2008, Bossé et al. 2014, Ito 2010, Jessing et al. 2003, Lo et al. 1998, Schuchert et al. 2004, Turni et al. 2014, Zhou et al. 2008). These PCR typing methods facilitate the reliable typing of A.pleuropneumoniae serovars without cross-reactions and mistyping. In the present study, we developed a multiplex PCR method for cps typing of A. pleuropneumoniae serovars 1, 2, 5, and 7, which are the most prevalent in Japan (Asawa et al. 1995, Fukuyasu et al. 1991, 1996, Kume \& Nakai 1988, Morioka et al. 2006, 2008, Suzuki et al. 1989, Yoshimura et al. 2002), as well as serovar 15, isolated cases of which have been increasing (Ito 2013, Morioka et al. 2008).

\section{Materials and methods}

We used the A.pleuropneumoniae serovar reference strains serovar 1, 4074; serovar 2, S1536; serovar 3, S1421; serovar 4, M62; serovar 5a, K17; serovar 6, Femø, serovar 7, WF83, serovar 8, 405; serovar 9; CVJ13261; serovar 10, D13039; serovar 11; 56153; serovar 12, 8329; serovar 13, N273; serovar 14, 4906; and serovar 15, HS143, to determine suitable conditions for multiplex PCR. Seventy-

* Corresponding author: e-mail itohiroy@affrc.go.jp

Received 7 October 2014; accepted 24 December 2014. 
eight Japanese field strains of A. pleuropneumoniae serovar $1(\mathrm{n}=15)$, serovar $2(\mathrm{n}=15)$, serovar $5(\mathrm{n}=15)$, serovar 7 $(\mathrm{n}=13)$, serovar $8(\mathrm{n}=3)$, serovar $12(\mathrm{n}=3)$, and serovar 15 $(n=12)$, as well as Canadian serovar 10 strains $(n=2)$ were examined to assess the feasibility of using our new method. These strains were isolated from various areas and over several decades (from 1976 to 2011). The organisms were cultivated on TSA agar (Difco, Sparks, MD) supplemented with $5 \%$ defibrinated horse blood and $2 \%$ fresh yeast extract at $37^{\circ} \mathrm{C}$.

Template DNAs of the serovar references and field strains were extracted with the High Pure PCR Template Preparation Kit and High Pure PCR Product Purification Kit (Roche Diagnostics, Mannheim, Germany). Multiplex PCR was performed in a total volume of $50 \mu \mathrm{L}$ containing $1 \times$ buffer (Toyobo, Tokyo), $0.2 \mathrm{mM}$ of each dNTP, $0.3 \mu \mathrm{M}$ of each serovar- and species-specific primer (Table 1), 2.5 units of KOD FX Neo (Toyobo, Tokyo), and $10 \mathrm{ng}$ of template DNA. The serovar 1, 2, 5, and 7- specific primers (Angen et al. 2008, Jessing et al. 2003, Lo et al. 1998) and species-specific primers (Gram \& Ahrens 1998) used in this study were previously reported (Table 1). New primers for serovar 15 were also designed based on the nucleotide sequence of the cps $15 \mathrm{C}$ gene (Accession number: AB701753), which has been reported as specific to serovar 15 (Ito \& Sueyoshi 2015). The amplification steps used were as follows: $94^{\circ} \mathrm{C}$ for 2 min (preheating); 35 cycles of $98^{\circ} \mathrm{C}$ for $10 \mathrm{sec}$ (heat denature), $64^{\circ} \mathrm{C}$ for $30 \mathrm{sec}$ (annealing), and $68^{\circ} \mathrm{C}$ for $1 \mathrm{~min}$ (extension); and a final step at $68^{\circ} \mathrm{C}$ for $5 \mathrm{~min}$. Five $\mu \mathrm{L}$ of each reaction was analyzed by electrophoresis with a $2 \%$ agarose gel.

\section{Results}

The reference strains of $A$. pleuropneumoniae serovars $1,2,5,7$, and 15 produced 0.75-, 0.5-, 1.1-, 0.4- and 0.2-kb PCR products, respectively (Fig. 1). In contrast, the reference strains of serovars 3, 4, 6, and 8-14 did not yield any serovar 1-, 2-, 5-, 7- or 15- specific PCR products (Fig. 1). All serovar reference strains produced $0.95-\mathrm{kb}$ A. pleuropneumoniae-specific amplicons (Fig. 1).

The field strains of A. pleuropneumoniae serovars 1, 2, 5, 7, and 15 produced 0.75-, 0.5-, 1.1-, 0.4- and 0.2-kb PCR products, respectively (data not shown) as did the

Table 1. Primers used for PCR amplification

\begin{tabular}{|c|c|c|c|c|c|}
\hline $\begin{array}{l}\text { Primer } \\
\text { name }\end{array}$ & Function & Sequence & $\begin{array}{l}\text { Amplicon size } \\
\text { (kilobase pairs) }\end{array}$ & DNA amplified & References \\
\hline Ap1F & $\begin{array}{l}\text { Forward primer for } \\
\text { cps region of serovar } 1\end{array}$ & GGG CAA GCC TCT GCT CGT AA & 0.75 & serovar 1 & Angen et al. 2008 \\
\hline Ap1R & $\begin{array}{l}\text { Reverse primer for } \\
\text { cps region of serovar } 1\end{array}$ & GAA AGA ACC AAG CTC CTG CAA T & & & Angen et al. 2008 \\
\hline Ap2F & $\begin{array}{l}\text { Forward primer for } \\
\text { cps region of serovar } 2\end{array}$ & ACT ATG GCA ATC AGT CGA TTC AT & 0.5 & serovar 2 & Jessing et al. 2003 \\
\hline Ap2R & $\begin{array}{l}\text { Reverse primer for } \\
\text { cps region of serovar } 2\end{array}$ & CCT AAT CGG AAA CGC CAT TCT G & & & Jessing et al. 2003 \\
\hline Ap5A & $\begin{array}{l}\text { Forward primer for } \\
\text { cps region of serovar } 5\end{array}$ & TTT ATC ACT ATC ACC GTC CAC ACC T & 1.1 & serovar 5 & Lo et al. 1998 \\
\hline Ap5B & $\begin{array}{l}\text { Reverse primer for } \\
\text { cps region of serovar } 5\end{array}$ & CAT TCG GGT CTT GTG GCT ACT AAA & & & Lo et al. 1998 \\
\hline Ap7F & $\begin{array}{l}\text { Forward primer for } \\
\text { cps region of serovar } 7\end{array}$ & GGT GAC TGG CGT ACG CCA AA & 0.4 & serovar 7 & Angen et al. 2008 \\
\hline Ap7R & $\begin{array}{l}\text { Reverse primer for } \\
\text { cps region of serovar } 7\end{array}$ & GGG CTG CAG ACT GAC GTA A & & & Angen et al. 2008 \\
\hline Ap15F & $\begin{array}{l}\text { Forward primer for } \\
\text { cps region of serovar } 15\end{array}$ & GCA GAT TTG GGA TTC CGA TTT CGG & 0.2 & serovar 15 & This study \\
\hline Ap15R & $\begin{array}{l}\text { Reverse primer for } \\
\text { cps region of serovar } 15\end{array}$ & AGC AAC TTC AAT AAT CTG ACG ACG & & & This study \\
\hline LPF & $\begin{array}{l}\text { Forward primer for } \\
\text { omlA gene }\end{array}$ & AAG GTT GAT ATG TCC GCA CC & 0.95 & serovars 1 to 15 & $\begin{array}{l}\text { Gram \& Ahrens } \\
1998\end{array}$ \\
\hline LPR & $\begin{array}{l}\text { Reverse primer for } \\
\text { omlA gene }\end{array}$ & CAC CGA TTA CGC CTT GCC A & & & $\begin{array}{l}\text { Gram \& Ahrens } \\
1998\end{array}$ \\
\hline
\end{tabular}




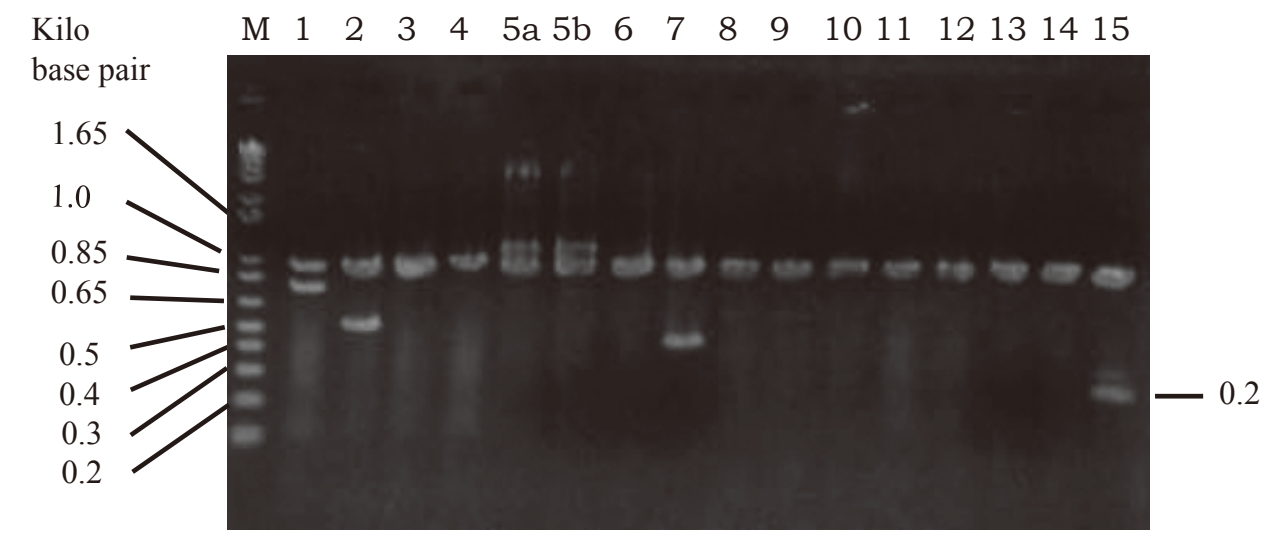

Fig. 1. Multiplex PCR results with serovar reference strains. The number above each lane indicates the serovar. M: 1 kb plus DNA ladder (Invtirogen, Carlsbad, CA).

serovar reference strains. The field strains of serovars 8,10 , and 12 yielded faint PCR products with a band size slightly exceeding that amplified from the serovar 2 strain, although these field strains did not yield any serovar 1-, 2-, 5-, 7- or 15-specific PCR products. However, each faint DNA band could be easily discriminated from the intense bands from serovars 1, 2, 5, 7, and 15. All field strains produced 0.95$\mathrm{kb}$ species-specific amplicons.

\section{Discussion}

In the present study, we focused on developing a multiplex PCR method for cps typing of $A$. pleuropneumoniae serovars $1,2,5$, and 7 because they are the most predominant serovars in Japan, accounting for approx. 98\%, as well as the newly identified serovar 15 , since its incidence in the form of isolated cases has recently been increasing. This multiplex PCR method may be useful, not only in Japan, but also worldwide, since serovars 1, 5, and 7 in North America (Gottschalk 2012), serovar 2 in most of Europe (Gottschalk 2012), and serovar 15 in Australia (Blackall et al. 2002) are the most dominant serovars causing most outbreaks in these regions. In conclusion, the present multiplex PCR method for serovars $1,2,5,7$, and 15 may be used as a valuable tool complementing the serotyping of $A$. pleuropneumoniae field strains for diagnoses and epidemiological studies.

\section{Acknowledgements}

This study was supported by grants from the National Agriculture and Food Research Organization, Japan.

\section{References}

Angen, Ø. et al. (2008) Development of a multiplex PCR test for identification of Actinobacillus pleuropneumoniae serovar 1, 7, and 12. Vet. Microbiol., 132, 312-318.

Asawa, T. et al. (1995) Serotypes and antimicrobial susceptibility of Actinobacillus pleuropneumoniae isolated from piglets with pleuropneumonia. J. Vet. Med. Sci., 57, 757-759.

Blackall, P. J. et al. (2002) Proposal of a new serovar Actinobacillus pleuropneumoniae: serovar 15. Vet. Microbiol., 84, 47-52.

Bossé, J. T. et al. (2014) Multiplex PCR assay for unequivocal differentiation of Actinobacillus pleuropneumoniae serovars 1 to 3, 5 to 8, 10, and 12. J. Clin. Microbiol., 52, 2380-2385.

Fukuyasu, T. et al. (1991) Serotyping and drug susceptibility of strains of Actinobacillus (Haemophilus) pleuropneumoniae isolated from pneumonic lungs of pigs. J. Jpn. Vet. Med. Assoc., 44, 11-16 [In Japanese with English summary].

Fukuyasu, T. et al. (1996) Serotypes and antimicrobial susceptibility of Actinobacillus pleuropneumoniae isolated from pigs with pneumonia. J. Jpn. Vet. Med. Assoc., 49, 528-532 [In Japanese with English summary].

Gottschalk, M. (2012) Diseases of swine, 10th ed. Wiley-Blackwell, Oxford, UK, 653-669.

Gram, T. \& Ahrens, P. (1998) Improved diagnostic PCR assay for Actinobacillus pleuropneumoniae based on the nucleotide sequence of an outer membrane lipoprotein. J. Clin. Microbiol., 36, 443-448.

Ito, H. (2010) Development of a cps-based multiplex PCR for typing of Actinobacillus pleuropneumoniae serotypes 1, 2 and 5. J. Vet. Med. Sci., 72, 653-655.

Ito, H. (2013) Recent topics of serotypes/serotyping and host specificity of Actinobacillus pleuropneumoniae. Proc. Jpn. Pig Vet. Soc., 61, 14-21 [In Japanese].

Ito, H. \& Sueyoshi, M. (2015) The genetic organization of the capsular polysaccharide biosynthesis region of Actinobacillus pleuropneumoniae serotype 15. J. Vet. Med. Sci., 77, 483486. 
Jessing, S. G. et al. (2003) Evaluation of a multiplex PCR test for simulutaneous identifiaction and serotyping of Actinobacillus pleuropneumoniae serotypes 2, 5, and 6. J. Clin.Microbiol., 41, 4095-4100.

Koyama, T. et al. (2007) Isolation of Actinobacillus pleuropneumoniae serovar 15-like strain from a field case of porcine pleuropneumonia in Japan. J. Vet. Med. Sci., 69, 961-964.

Kume, K. \& Nakai, T. (1988) Isolation of Actinobacillus (Haemophilus) pleuropneumoniae serovar 1, 6, or 7 from pigs. Jpn. J. Vet. Sci., 50, 589-591.

Lo, T. M. et al. (1998) Detection and identification of Actinobacillus pleuropneumoniae serotype 5 by multiplex PCR. $J$. Clin. Microbiol., 36, 1704-1710.

Morioka, A. et al. (2006) Antimicrobial susceptibility of Actinobacillus pleuropneumoniae isolates during 1999 and 2000 in Japan. J. Jpn. Vet. Med. Assoc., 59, 815-819 [In Japanese with English summary].

Morioka, A. et al. (2008) Recent trends in antimicrobial susceptibility and the presence of the tetracycline resistance gene in Actinobacillus pleuropneumoniae isolates in Japan. J. Vet. Med. Sci., 70, 1261-1264.
Perry, M. B. et al. (1990) Structural characteristics of the antigenic capsular polysaccharides and lipopolysaccharides involved in the serological classification of Actinobacillus (Haemophilus) pleuropneumoniae strains. Serodiagn. Immunother. Inf. Dis., 4, 299-308.

Schuchert, J. A. et al. (2004) Detection and identification of Actinobacillus pleuropneumoniae serotypes 1, 2, and 8 by multiplex PCR. J. Clin. Microbiol., 42, 4344-4348.

Suzuki, S. et al. (1989) Antimicrobial susceptibility of Actinobacillus (Haemophilus) pleuropneumoniae isolated from pigs with pleuropneumonia. Jpn. J. Vet. Sci., 51, 450-452.

Turni, C. et al. (2014) Evaluation of a multiplex PCR to identify and serotype Actinobacillus pleuropneumoniae serovars 1, 5, 7, 12 and 15. Lett. Appl. Microbiol., 59, 362-369.

Yoshimura, H. et al. (2002) Comparative in vitro activity of 16 antimicrobial agents against Actinobacillus pleuropneumoniae. Vet. Res. Commun., 26, 11-19.

Zhou, L. et al. (2008) Multiplex PCR that can distinguish between immunologically cross-reactive serovar 3, 6, and 8 Actinobacillus pleuropneumoniae strains. J. Clin. Microbiol., 46, 800-803. 